\title{
Risk assessment of malaria transmission at the border area of China and Myanmar
}

\author{
Benyun Shi ${ }^{1 *}$, Jinxin Zheng ${ }^{2}$, Hongjun Qiu' ${ }^{1}$ Guo-Jing Yang ${ }^{2 *}$, Shang Xia ${ }^{3}$ and Xiao-Nong Zhou ${ }^{3,4}$
}

\begin{abstract}
Background: In order to achieve the goal of malaria elimination, the Chinese government launched the National Malaria Elimination Programme in 2010. However, as a result of increasing cross-border population movements, the risk of imported malaria cases still exists at the border areas of China, resulting in a potential threat of local transmission. The focus of this paper is to assess the Plasmodium vivax incidences in Tengchong, Yunnan Province, at the border areas of China and Myanmar.

Methods: Time series of P. vivax incidences in Tengchong from 2006 to 2010 are collected from the web-based China Information System for Disease Control and Prevention, which are further separated into time series of imported and local cases. First, the seasonal and trend decomposition are performed on time series of imported cases using Loess method. Then, the impact of climatic factors on the local transmission of $P$. vivax is assessed using both linear regression models (LRM) and generalized additive models (GAM). Specifically, the notion of vectorial capacity (VCAP) is used to estimate the transmission potential of $P$. vivax at different locations, which is calculated based on temperature and rainfall collected from China Meteorological Administration.

Results: Comparing with Ruili County, the seasonal pattern of imported cases in Tengchong is different: Tengchong has only one peak, while Ruili has two peaks during each year. This may be due to the different cross-border behaviors of peoples in two locations. The vectorial capacity together with the imported cases and the average humidity, can well explain the local incidences of $P$. vivax through both LRM and GAM methods. Moreover, the maximum daily temperature is verified to be more suitable to calculate VCAP than the minimal and average temperature in Tengchong County.

Conclusion: To achieve malaria elimination in China, the assessment results in this paper will provide further guidance in active surveillance and control of malaria at the border areas of China and Myanmar.
\end{abstract}

\section{Multilingual abstracts}

Please see Additional file 1 for translation of the abstract into the five official working languages of the United Nations.

\section{Background}

Malaria is one of the important vector-borne infectious diseases that seriously endanger the public health and affect the socio-economic development $[1,2]$. For example, malaria caused approximately 216 million infections and about 438000 deaths worldwide in 2015 [3]. Many

*Correspondence: benyunshi@outlook.com; guojingyang@hotmail.com 'School of Cyberspace, Hangzhou Dianzi University, 310018 Hangzhou, China ${ }^{2}$ Jiangsu Institute of Parasitic Diseases, 214064 Wuxi, China

Full list of author information is available at the end of the article initiatives and programmes have been launched to help develop implementable action plans for malaria control and elimination, such as the Global Malaria Initiatives, the U.S. President's Malaria Initiative and the Lubombo Spatial Development Initiative [4]. Previous experiences emphasize that border areas should be a focus for malaria control activities given the high intensity of both formal and informal movement of people and goods across borders [5]. For exmaple, in the Greater Mekong Subregion, malaria transmission is largely confined to the border areas between Burma, Cambodia, and Thailand [6]. On the one hand, the cross-border movement of populations can introduce malaria cases from high-transmission areas into previously low-transmission or malaria-free areas [7-10]. On the other hand, the imported cases may cause 
the recurrence of malaria when the environment is suitable for local transmission. Therefore, to achieve global malaria eradication, one of the most important research agenda is to strengthen regional intercountry collaborations, especially the cross-border collaborations [11].

Historically, malaria was extensively endemic in China, especially in the 1960s and 1970s [12]. Since the Chinese government launched the National Malaria Elimination Programme (NMEP) in 2010, great progress have been made to achieve the elimination of malaria. During the implementation of the NMEP from 2010 to 2014, local malaria incidence has declined continuously, except for the border areas of Yunnan Province, China [13]. Yunnan Province shares a 4 061-kilometer borderline with Myanmar, Laos, and Vietnam. Because the border areas are mostly mountainous, crossing the border becomes very easy. It has been estimated that there are several millions of people belonging to the mobile and migrant population (MMP), who may cross the border every year [14]. In 2011, the number of malaria cases in Yunnan Province was the highest in China and accounted for $34 \%$ of the total cases in the country [15]. However, according to the malaria report in 2013, about 91.2\% malaria cases in Yunnan Province are imported from neighboring countries, among which $79.6 \%$ were caused by the Plasmodium vivax parasite [16]. Because the imported cases can result in local transmission of malaria in a suitable environment, it becomes one of the biggest obstacles for nationwide elimination of the disease in China $[17,18]$.

In this paper, we focus on assessing the transmission risk of $P$. vivax in Tengchong County, Yunnan Province, China, which is at the border area of China and Myanmar. Existing studies have shown that the impedance of malaria control in Tengchong are more likely due to the increase of formal and informal human movement across China-Myanmar border regions $[19,20]$. Different crossborder activities may result in different temporal patterns of imported cases (e.g., the number of peak transmission seasons). For example, people engaged in frontier trade may frequently cross the border; while local farmers may go to Myanmar for logging or mining during the slack seasons in farming. In this case, to take a step forward to malaria elimination and prevent the recurrence of malaria in China, it would be better to analyze the temporal patterns of imported cases so as to further investigate the purpose of human cross-border activities. By doing so, active surveillance and target interventions can be planned and implemented.

From the perspective of disease epidemiology, the natural transmission of $P$. vivax depends on the interactions between female anopheles mosquitoes and human beings. Existing studies have shown that the ability of mosquitoes to transmit $P$. vivax can be affected by a series of biological factors, such as the daily survival rate of mosquitoes and the sporogonic cycle of sporozoits in their bodies [21]. Researchers have revealed that meteorological factors can also significantly affect mosquito population as well as their biological cycles [22, 23]. Specifically, a vectorial capacity model has been proposed to estimate the malaria transmission potential, which takes into consideration the impact of temperature and rainfall on the bionomics of mosquitoes and the extrinsic incubation period of parasites [24]. Taking into consideration both human cross-border movements and local environmental factors, the objective of this work is twofold: (i) to investigate the temporal patterns of imported cases so as to guide the implementation of active surveillance; and (ii) to evaluate the impact of meteorological factors on the local transmission risk of $P$. vivax in Tengchong County.

The analysis procedures of this paper is organized as follows. First, we demonstrate the different temporal patterns of imported $P$. vivax cases in Tengchong and Ruili counties by performing seasonal and trend decomposition using Loess method. Then, we assess the relationship between local $P$. vivax cases and meteorological factors using both linear regression models (LRM) and generalized additive models (GAM). Moreover, to characterize the nonlinear relationship between $P$. vivax transmission potential and meteorological factors, the notion of vectorial capacity (VCAP) is adopted, which is defined as "the number of potentially infective contacts an individual person makes, through vector population, per unit time [25]." Both the LRM and GAM results show that the VCAP together with the imported cases can better explained the local infections of $P$. vivax. Specifically, we verify that the maximum daily temperature is more suitable to calculate VCAP than the minimal and average temperature in Tengchong County.

\section{Methods}

\section{Data collection}

In China, there is a sound surveillance system for infectious diseases, namely the China Information System for Disease Control and Prevention (CISDCP), where malaria cases are reported daily from each public health facility [26]. According to the Action Plan of China Malaria Elimination, it is obligatory for any medical institutions and hospitals to report clinically confirmed infection cases into the system. In this case, even though the underreporting of $P$. vivax infections is still unavoidable, the number of missing report should be negligible. Time series of $P$. vivax cases in Tengchong and Ruili are collected and aggregated from CISDCP on a daily basis ranging from 2006 to 2010, where imported cases $\left(I_{P . v}\right)$ are discriminated from local infections $\left(L_{P . v}\right)$ by doctors or public health investigators through face-to-face case studies. Accordingly, meteorological data are collected from China Meteorological Administration on a daily basis, which include 
the minimum temperature $\left(T_{\min }\right)$, average temperature $\left(T_{\text {avg }}\right)$, maximum temperature $\left(T_{\max }\right)$, rainfall $(R)$, average humidity $\left(H_{\text {avg }}\right)$, and minimum humidity $\left(H_{\text {min }}\right)$.

\section{Vectorial capacity}

The notion of vectorial capacity (VCAP) has been extensively used to assess malaria transmission potential based on meteorological factors $[25,27,28]$. The VCAP is derived from the basic reproductive number calculated based on the Macdonald model [29]. Mathematically, it is formulated as follows:

$$
V=\frac{-\left(m a^{2}\right) p^{n}}{\ln (p)}
$$

where $m$ represents the equilibrium mosquito density per person, $a$ is the expected number of bites on human beings per mosquito per day, $p$ is the probability of a mosquito surviving through one whole day, and $n$ is the entomological incubation period of malaria parasites. Based on the study of Ceccato et al. in [24], all these parameters are dynamically dependent on the temperature $T$ and rainfall $R$. Specifically, we have $m=100 * R$, $a=0.7 / g, g=[36.5 /(T+2.0-9.9)]+0.5, p=0.5^{1 / g}$, and $n=105 / 2 *[36.5 /(T+2.0-9.9)] / g+T-18$. For more details about the model parameters, please refer to the Table 2 in [27]. With respect to different temperature (resp., $T_{\min }, T_{a v g}$, and $T_{\max }$ ), we have different values of VCAP (resp., $V_{\text {min }}, V_{a v g}$, and $V_{\text {max }}$ ), where the subscripts correspond to temperature $T_{\min }, T_{a v g}$, and $T_{\max }$. In this paper, we exam the suitability of $T_{\min }, T_{a v g}$, and $T_{\max }$ to estimate the transmission potential of $P$. vivax in Tengchong County.

\section{Analysis methods}

To reveal the temporal patterns of imported cases, the seasonal and trend decomposition are performed on the daily time series using Loess method. To a certain extent, the seasonality reveals the pattern of human cross-border movements, which can guide the active surveillance and control of imported cases from neighboring countries. While the trend reflects the strength of disease intervention implemented by public health authorities, which are further adopted in our methods to model the risk of local malaria transmission.

To explore the relationship between local transmission risk of $P$. vivax and associated environmental factors, time series of local P. vivax incidences in Tengchong are fitted by meteorological factors using both linear regression models (LRM) and generalized additive models (GAM). Since the number of daily $P$. vivax incidences are very small, in this study, both imported and local cases are aggregated biweekly. Accordingly, the time series of environmental factors are also averaged over 14 days. This is reasonable because although the $P$. vivax parasites may stay dormant for a long time, the incubation period of $P$. vivax is usually from 12 to 20 days. The Pearson coefficient is first calculated to measure the linear correlation between each pair of these meteorological factors. Then, several LRM and GAM models are assessed to predict the expected number of local incidences $\left(L_{P . v}\right)$ from the meteorological factors and the number of imported cases $\left(I_{P . v}\right)$. Mathematically, the following models are proposed:

$$
\begin{aligned}
\text { LRM-1:L } L_{P . v}=\beta_{0}+ & \beta_{1} T_{\text {min }}+\beta_{2} T_{a v g}+\beta_{3} T_{\text {max }} \\
+ & \beta_{4} R+\beta_{5} H_{a v g}+\beta_{6} H_{\text {min }}+\beta_{7} I_{P . v}, \\
\text { LRM-2:L } L_{P . v}=\beta_{0}+ & \beta_{1} T_{\text {avg }}+\beta_{2} R+\beta_{3} H_{\text {avg }}+\beta_{4} I_{P . v}, \\
\text { GAM-1: } \log \left(L_{P . v}\right)= & \beta_{0}+f_{1}\left(T_{\text {min }}\right)+f_{2}\left(T_{\text {avg }}\right) \\
& +f_{3}\left(T_{\text {max }}\right)+f_{4}(R)+f_{5}\left(H_{\text {avg }}\right) \\
& +f_{6}\left(H_{\text {min }}\right)+f_{7}\left(I_{P . v}\right), \\
\text { GAM-2: } \log \left(L_{P . v}\right)= & \beta_{0}+f_{1}\left(T_{\text {avg }}\right)+f_{2}(R)+f_{3}\left(H_{\text {avg }}\right) \\
& +f_{4}\left(I_{P . v}\right),
\end{aligned}
$$

The reason to propose LRM-2 and GAM-2 is that there exists significant correlation among $T_{\min }, T_{a v g}$, and $T_{\max }$, and between $H_{\text {min }}$ and $H_{\text {avg }}$.

In addition, another three assessment models are built upon the notion of vectorial capacity $V$, which integrates both temperature and rainfall. Taking into consideration the strength of malaria control implemented by public health authorities, a linear trend of time series of $P$. vivax incidences is combined with VCAP to reflect the risk of $P$. vivax infection. To assess the suitability of $V_{\text {min }}, V_{\text {avg }}$, and $V_{\text {max }}$ for estimating the $P$. vivax transmission potential, the following GAM models are proposed:

$$
\begin{aligned}
\operatorname{GAM}-\mathrm{V}-\mathrm{MIN}: \log \left(L_{P . v}\right)= & \beta_{0}+f_{1}\left((a+b t) V_{\text {min }}\right) \\
& +f_{2}\left(H_{\text {avg }}\right)+f_{3}\left(I_{P . v}\right), \\
\text { GAM-V-AVG: } \log \left(L_{P . v}\right)= & \beta_{0}+f_{1}\left((a+b t) V_{a v g}\right) \\
& +f_{2}\left(H_{a v g}\right)+f_{3}\left(I_{P . v}\right), \\
\text { GAM-V-MAX: } \log \left(L_{P . v}\right)= & \beta_{0}+f_{1}\left((a+b t) V_{\text {max }}\right) \\
& +f_{2}\left(H_{\text {avg }}\right)+f_{3}\left(I_{P . v}\right) .
\end{aligned}
$$

where $(a+b t)$ is the trend obtained from the seasonal and trend decomposition of P. vivax incidences. All these models will be assessed based on the available dataset in Tengchong County.

\section{Results}

Time series decomposition of $P$. vivax cases

The seasonal and trend decomposition are performed on time series of imported $P$. vivax cases using Loess method. To a certain extent, the seasonality reveals the pattern of 
human cross-border movements, while the trend reflects the strength of malaria intervention and control. A comparison is conducted between Tengchong and Ruili, where Ruili is another malaria-endemic county at the border area of China and Myanmar. Figure 1 shows the decomposition results in Tengchong County, and Fig. 2 shows the decomposition results in Ruili County. It can be observed that the trend of imported P. vivax cases in both counties declines almost linearly, reflecting the effectiveness of the implemented malaria intervention policy. However, the patterns of seasonality is totally different in the two counties: Tengchong has only one peak every year, while Ruili has two peaks. This may be due to different human cross-border activities in these two counties. Comparing to Ruili, Tengchong is a little far away from the customs on the China-Myanmar border. Detailed investigations in Tengchong show that most farmers in the same villages often go to Myanmar in groups during the slack season, and come back before the busy seasons. Such activities happens once a year. However, due to the lack of manpower and resources, such investigations in Ruili are not put into effect. Even so, the observations provide an insight into the implementation of active surveillance on imported cases to further investigate the motivations of their cross-border activities other counties at the border area. The reason is that different cross-border activities may result in different seasonal patterns of imported cases, which further trigger different risks of infection at different time of a year. Therefore, targeted intervention strategies are required for counties with different patterns of imported cases.

\section{The relationship between local infections and meteorological factors}

To reveal the relationship between local infections and meteorological factors, the Pearson correlation coefficients between each pair of meteorological factors are first calculated, where daily time series of $T_{\text {min }}, T_{\text {avg }}, T_{\text {max }}$, $R, H_{\text {avg }}$ and $H_{\text {min }}$ with lag zero are used. Table 1 shows the correlation matrix between meteorological factors in Tengchong County. Strong linear correlation can be found among $T_{\text {min }}, T_{a v g}$, and $T_{\max }$, as well as between $H_{a v g}$ and $H_{\text {min }}$. In this case, only two types of LRM models (i.e., LRM-1 and LRM-2) and two types of GAM models (GAM-1 and GAM-2) are compared to assess time series of local P.vivax infections. Given a set of candidate models for the data, the preferred model is the one with the minimum Akaike information criterion (AIC) and Bayesian information criterion (BIC) values. Table 2 shows the comparisons of LRM and GAM models with respect to local infections of P.vivax in Tengchong County. First, it can be observed that the nonlinear GAM models have better performances than corresponding LRM models. For example, GAM-1 (resp., GAM-2) model is better than LRM-1 (resp., LRM-2) model with smaller values of $\mathrm{AIC}, \mathrm{BIC}$ and mean squared error (MSE), and larger $R^{2}$ and percentage of deviance explain. Second, even though some meteorological factors have strong correlations, the LRM-1 (resp., GAM-1) model, which involves more meteorological factors, has smaller AIC value but larger BIC value than the LRM-2 (resp., GAM-2) model. when fitting models, more parameters may result in overfitting. Both AIC and BIC rewards goodness of fit, but they also

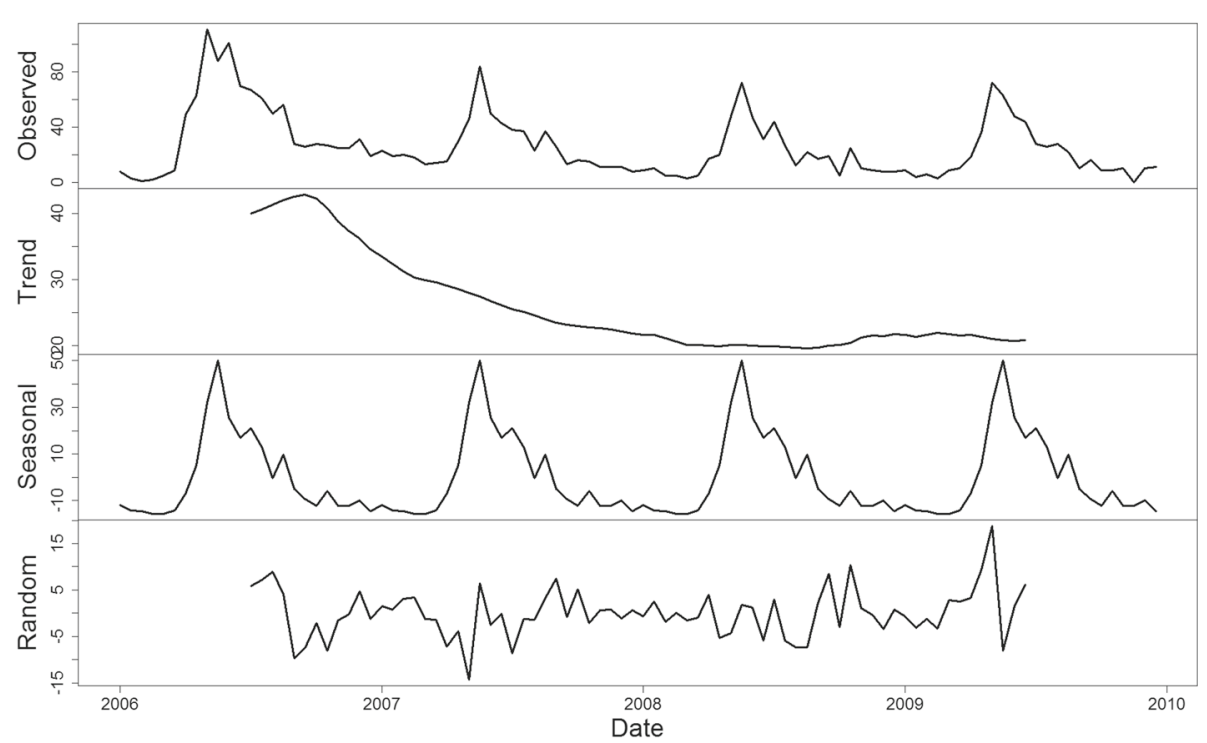

Fig. 1 The seasonal and trend decomposition of imported vivax malaria cases in Tengchong Countiy. The seasonality reveals the pattern of human cross-border movements, while the trend reflects the strength of malaria intervention and control. It can be observed that there is only one peak of imported cases each year 


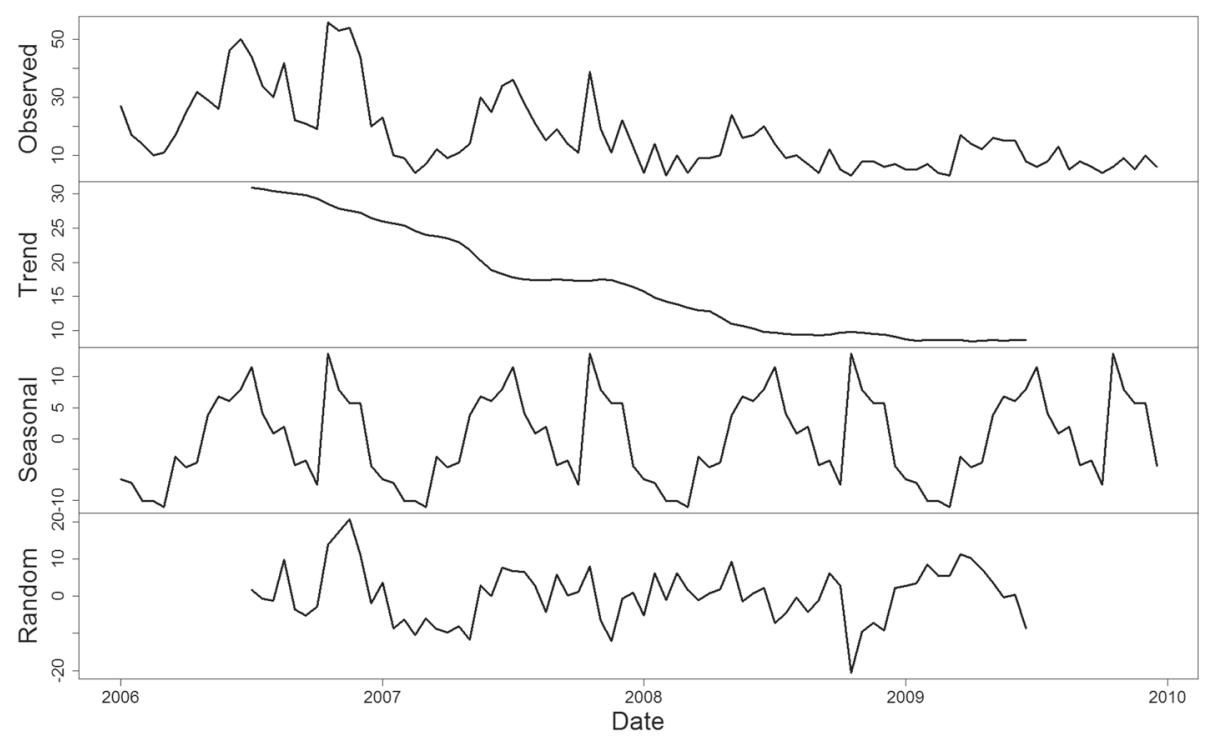

Fig. 2 The seasonal and trend decomposition of imported vivax malaria cases in Ruili County. The seasonality reveals the pattern of human cross-border movements, while the trend reflects the strength of malaria intervention and control. It can be observed that there are two peaks of imported cases each year

attempt to resolve the overfitting problem by introducing a penalty that is an increasing function of the number of estimated parameters (NUM). However, the penalty term in BIC is larger than in AIC. Therefore, we cannot determine which model between LRM-1 and LRM-2 (resp., between GAM-1 and GAM-2) is better.

\section{The GAM models with vectorial capacity}

Based on Eq. 1, the estimation of vectorial capacity $V$ involves both temperature $T$ and rainfall $R$ in Tengchong. Accordingly, three VCAP values $V_{\text {min }}$, $V_{\text {avg }}$, and $V_{\max }$ can be calculated using different temperatures $T_{\min }, T_{a v g}$, and $T_{\max }$ (see Fig. 3). It can be observed that the VCAP curves have similar temporal patterns to the time series of $P$. vivax incidences in Tengchong. Since GAM models is superior to LRM models (see Table 2), to evaluate the effects of VCAP on local $P$. vivax infections, only GAM models are used to conduct the comparison (i.e., the

Table 1 The Pearson coefficient matrix between time series of meteorological factors in Tengchong County

\begin{tabular}{lllllll}
\hline & $T_{\text {avg }}$ & $T_{\min }$ & $T_{\max }$ & $R$ & $H_{\text {avg }}$ & $H_{\min }$ \\
\hline$T_{\text {avg }}$ & 1 & & & & & \\
$T_{\min }$ & 0.98 & 1 & & & & \\
$T_{\max }$ & 0.94 & 0.86 & 1 & & & \\
$R$ & 0.662 & 0.73 & 0.47 & 1 & & \\
$H_{\text {avg }}$ & 0.63 & 0.75 & 0.36 & 0.72 & 1 & \\
$H_{\text {min }}$ & 0.72 & 0.83 & 0.45 & 0.80 & 0.96 & 1 \\
\hline
\end{tabular}

GAM-V-MIN, GAM-V-AVG, and GAM-V-MAX models), where a linear trend $(a+b t)$ is combined with VCAP to approximate the declining trend observed from time series decomposition in Tengchong County. Table 3 shows the performance of the three models with respect to local infections of P. vivax in Tengchong. Comparing with the LRM and GAM models in Table 2, all the three models have much better performance, representing that VCAP is more suitable to explain the risk of local infection. Moreover, the parametric coefficients show that all variables $V_{\min }, V_{\text {avg }}, V_{\max }, H_{a v g}$, and $I_{P . v}$ strongly support these models with significance level $p<0.01$.

\section{Model selection for estimating vectorial capacity}

The results in Table 3 can also determine which temperature among $T_{\min }, T_{a v g}$, and $T_{\max }$ is better for estimating the transmission potential of $P$. vivax in Tengchong. This is critical for the prediction of malaria outbreaks. It can be

Table 2 The comparison of LRM and GAM models with respect to local $P$. vivax infections

\begin{tabular}{lllllllll}
\hline Model & linear & df & NUM & AIC & BIC & MSE & R/squ & $\begin{array}{l}\text { Deviance } \\
\text { explain \% }\end{array}$ \\
\hline LRM-1 & Y & 9.00 & 8 & 208.22 & 231.30 & 0.43 & 0.34 & 33.80 \\
GAM-1 & N & 18.31 & 8 & 197.84 & 244.80 & 0.31 & 0.45 & 45.30 \\
LRM-2 & $Y$ & 6.00 & 5 & 211.53 & 226.92 & 0.47 & 0.29 & 29.40 \\
GAM-2 & N & 10.02 & 5 & 206.57 & 232.26 & 0.41 & 0.36 & 35.50 \\
\hline
\end{tabular}

$d f$ residual degrees-of-freedom, NUM the number of parameters in the model, AIC Akaike information criterion, BIC Bayesian information criterion, MSE Mean squared error, $R$ /squ the coefficient of determination $R^{2}$ 


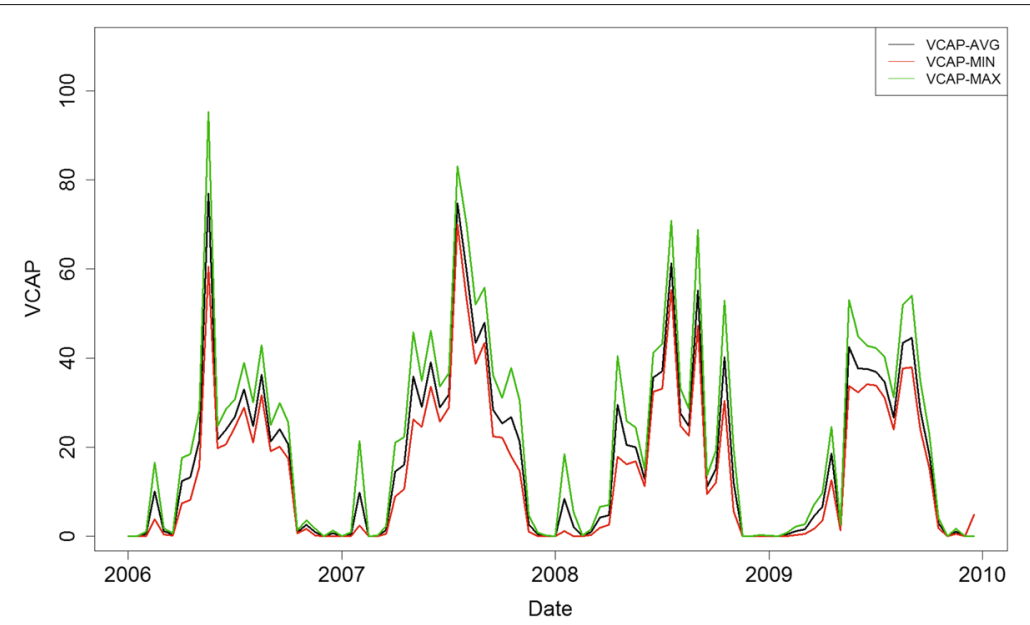

Fig. 3 The vectorial capacity calculated using various temperatures $T_{\min }, T_{\text {avg, }}$ and $T_{\max }$ in Tengchong County. The notion of vectorial capacity is defined as the number of potentially infective contacts an individual person makes, through vector population, per unit time

observed that the GAM-V-MAX model performs slightly better than the other two models. To further evaluate the model, we conduct the deviance analysis. The null hypothesis is that the three models fit the data equally well, and the alternative hypothesis is that the GAM-VMAX model is superior. Here the F-statistic is 4.34 and the associated $P$-value is 0.03 . The result provides very clear evidence that the GAM-V-MAX model involving maximum temperature is superior to the GAM-V-MIN and GAM-V-AVG models. Figure 4 shows the estimated curve of $P$. vivax infections based on the GAM-V-MAX model. It can be observed that most periods with high risk of infection can be estimated by the GAM-V-MAX model.

Figure 5 shows the estimated nonparametric smooths of VCAP, average humidity, and imported cases from the GAM-V-MAX model. Regions where the confidence bands (i.e., the dot lines) enclose the horizontal red line indicates corresponding values where the overall pattern is not significant. The smooth of imported cases indicate that the true relationship between the number of local infections and that of imported cases is linear. However, the estimated degrees of freedom (edf) for VCAP and average humidity is larger than 1 , which indicate possible

Table 3 The performance of VCAP-associated GAM models with respect to local $P$. vivax infections

\begin{tabular}{llllllllll}
\hline Model & linear df & Num & AIC & BIC & MSE & R/squ & $\begin{array}{l}\text { Deviance } \\
\text { explain \% }\end{array}$ \\
\hline GAM-V-AVG & $N$ & 7.84 & 4 & 143.83 & 161.70 & 0.34 & 0.455 & 50.0 \\
GAM-V-MIN & $N$ & 7.20 & 4 & 146.03 & 162.44 & 0.36 & 0.433 & 47.5 \\
GAM-V-MAX N & 8.18 & 4 & 141.87 & 160.51 & 0.33 & 0.472 & 51.8 \\
\hline
\end{tabular}

df residual degrees-of-freedom, NUM the number of parameters in the model, AIC Akaike information criterion, BIC Bayesian information criterion, MSE Mean squared error, $R /$ squ the coefficient of determination $R^{2}$ deviations from linearity. It can be observed from the Fig. 5 that the relationship with VCAP may be nearly linear: it appears that for lower VCAP the number of local infections increases at a constant rate but after passing a threshold value of VCAP the rate begins to decrease. On the other hand, the relationship with average humidity is not significant in most regions, except for the region between 60 and 70. In this region, the relationship with average humidity seems to be quadratic.

\section{Discussion}

Human population movement (HPM) have been cited amongst the significant causes of the failure of the Global Malaria Eradication Programme fifty years ago [30, 31]. Human population movement from high to low or non malaria-endemic areas can result in imported infections, which may further trigger onward transmission [7-10]. Moreover, HPM patterns and the risk of malaria transmission vary substantially across spatial and temporal scales, socioeconomic sub-groups, and motivation for travel. Therefore, strategic control and elimination planning requires quantitative information on HPM patterns and the translation of these into parasite dispersion [32]. Extensive studies have been conducted attempting to quantify HPM patterns. For example, Wesolowski et al. quantified the impact of human mobility on malaria in Kenya using mobile phone data [10]; Menach et al. investigated the travel risk and malaria importation between Zanzibar and mainland Tanzania using mobile phone data and ferry traffic [33]; Tatem et al. proposed a method for targeting of interventions using surveillance data, satellite imagery and mobile phone call records to support elimination planning in Namibia [34]; Pindolia et al. have investigated the demographics of human movement 


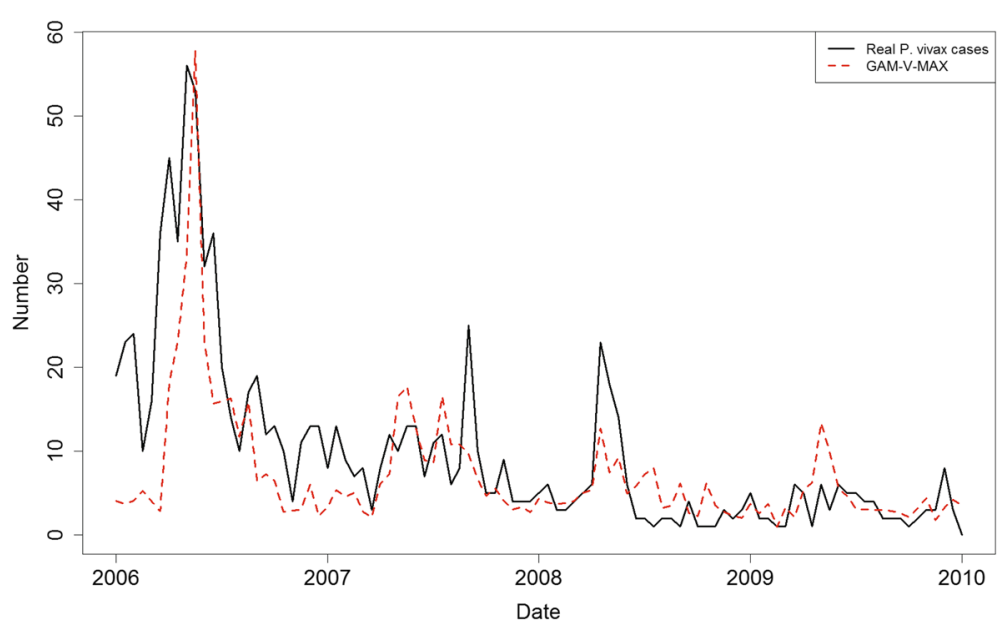

Fig. 4 The fitting result of GAM-V-MAX model with respect to the real number of $P$. vivax infections

and migration patterns in East Africa based on national population censuses and household surveys [35]. In high incidence areas of malaria, such census-style approaches have been verified to be helpful for malaria intervention and control. For China, which is about to reach the stage of malaria elimination and has only small number of imported cases, the above-mentioned approaches seem to be too resource-consuming and rough to characterize specific imported cases. In this paper, the seasonality analysis on time series of $P$. vivax incidences
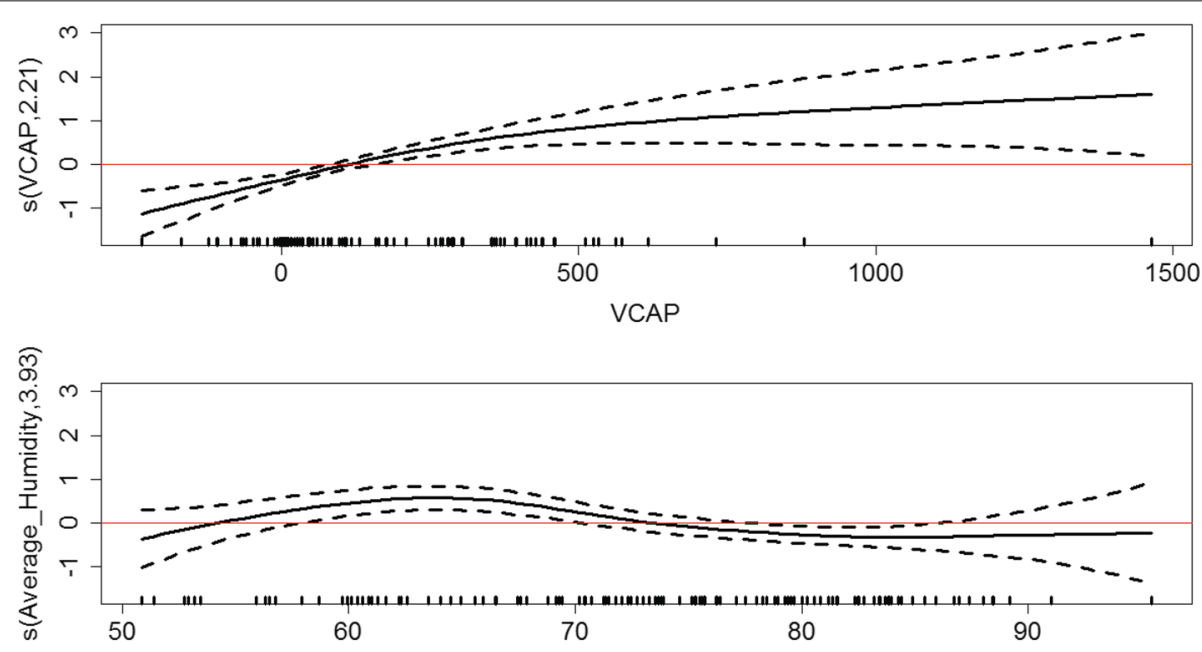

Average_Humidity

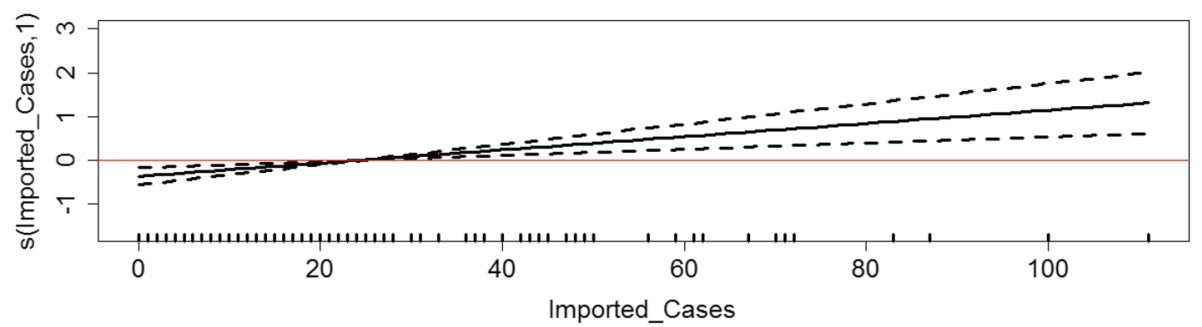

Fig. 5 The estimated nonparametric smooths of VCAP, average humidity, and imported cases from the GAM-V-MAX model. The smooth of imported cases indicates that the true relationship between the number of local infections and that of imported cases is linear. The smooth of VCAP indicates that for lower VCAP the number of local infections increases at a constant rate but after passing a threshold value of VCAP the rate begins to decrease. While the relationship with average humidity is not significant in most regions 
show that different patterns of human cross-border activity may result in different temporal patterns of $P$. vivax infections. Therefore, it would be helpful to perform active surveillance on motivations of human cross-border activity so as to plan targeted intervention strategies on imported cases.

Recently, together with the significant change of social and economic status, the corresponding malaria control strategies in Yunnan Province have also been changed. Besides traditional passive surveillance and vector controls, active surveillance and intervention have also been introduced, particularly in regions with high risk of infection. As compared with passive surveillance, active surveillance is much more ambitious with the aim of discovering every infection or imported case. Local CDC and surveillance agencies visit villages house by house to identify high risk populations. In this case, active surveillance is extremely time-consuming and requires massive experienced public health workers. However, human resources are very limited particularly in remote border area. For example, Tengchong has 18 towns, 205 villages and about 167964 households distributed in a wide area of 5845 square kilometers. However, in Tengchong CDC, less than 10 workers/investigators are available to perform active surveillance. The seasonality analysis for different counties can help frontier workers to perform active surveillance at the right place and time. For example, at Tengchong County, appropriate education and early warning can be conducted before peoples go to Myanmar once a year, while household survey can be implemented after they come back.

It is a particular challenge for malaria elimination at the China-Myanmar border area, especially when remote border areas of Myanmar have weak infrastructure and poor quality of treatments. In China, there is a sound surveillance system (CISDCP) for infectious diseases, where malaria incidences are reported from public health agencies everyday. However, in Myanmar, the surveillance and reporting system is too weak to collect sufficient information about the true malaria situation [36]. The weakness of health systems in Myanmar includes the limited capacity of local microscopists, incomplete coverage of surveillance for all communities and the lack of data reporting and management systems [19]. Because the meteorological factors are similar at the China-Myanmar border area, it is possible to use the same meteorological factors to assess the potential risk of malaria infection on both sides. In this paper, we have evaluated that the vectorial capacity $\left(V_{\max }\right)$ calculated by maximum temperature $\left(T_{\max }\right)$ and rainfall $(R)$ is more suitable to fit the local $P$. vivax incidences in Tengchong County. Without further improvement of health systems in Myanmar, the GAM-V-MAX model could temporarily be used to estimate the risk of infection at the border area. On the other hand, detailed case studies on imported cases in China can also provide valuable malaria situations in Myanmar from which they came back.

\section{Conclusion}

In this paper, we have assessed the risk of $P$. vivax transmission in Tengchong County, Yunnan Province, which is at the border area of China and Myanmar. First, different patterns of imported cases are decomposed for Tengchong and Ruili counties based on the seasonal and trend decomposition using Loess method. The seasonal patterns can provide useful information for active surveillance and further investigation of human cross-border movement, which is one of the biggest challenges for nationwide elimination of malaria in China. Second, the effect of meteorological factors on local risk of $P$. vivax infections is investigated using various linear regression models and generalized additive models. By comparison, it has been verified that the notion of vectorial capacity (VCAP) together with the number of imported cases is significant indicator to fit the number of local $P$. vivax incidences in Tengchong. Moreover, the maximum temperature has been assessed to be more suitable for estimating VCAP than the minimum and average temperature. Finally, the strong linear relationship with VCAP and imported cases indicates that to achieve effective malaria intervention, it is critical to perform active surveillance on the number of imported cases before the high risk season comes.

\section{Additional file}

Additional file 1: Multilingual abstracts in the five official working languages of the United Nations. (PDF $381 \mathrm{~kb}$ )

\section{Abbreviations}

CISDCP: China information system for disease control and prevention; GAM: Generalized additive model; HPM: Human population movement LRM: Linear regression model; MMP: Mobile and migrant population; NMEP: National malaria elimination programme; VCAP: Vectorial capacity

\section{Acknowledgements}

We would like to thank all of the study participants for their commitment. We also specifically thank Mr. Shengguo Li, Ms. Shouqin Yin, and other staff members at the Disease Prevention and Control Center of Tengchong County, Yunnan Province, China, for their efforts in data collection and investigation.

\section{Funding}

This work was supported by the National Natural Science Foundation of China (Grant Nos. 81402760, 81573261, 81502858, 81273192), the Natural Science Foundation of Jiangsu Province, China (Grant No. BK20161563), the Hong Kong Research Grants Council (RGC/HKBU12202415), and the Research Foundation of Education Bureau of Zhejiang Province, China (Grant No. Y201222907). The funders had no role in study design, data collection and analysis, decision to publish, or preparation of the manuscript.

Availability of data and materials

Please contact author for data requests. 


\section{Authors' contributions}

$B S, G Y, S X$, and XZ designed research, BS, SX and XZ collected the data, BS, JZ, $G Y, H Q$, and $S X$ conceived the experiments, BS and JZ conducted the experiments, $B S, J Z, G Y$, and $H Q$ analysed the results. BS wrote the manuscript and all authors reviewed the manuscript. All authors read and approved the final manuscript.

\section{Competing interests}

The authors declare that they have no competing interests.

\section{Consent for publication}

Not applicable.

\section{Ethics approval and consent to participate}

Not applicable.

\section{Author details}

${ }^{1}$ School of Cyberspace, Hangzhou Dianzi University, 310018 Hangzhou, China. ${ }^{2}$ Jiangsu Institute of Parasitic Diseases, 214064 Wuxi, China. ${ }^{3}$ National Institute of Parasitic Diseases, Chinese Center for Disease Control and Prevention, Shanghai, China. ${ }^{4}$ Key Laboratory of Parasite and Vector Biology, $\mathrm{MOH} ; \mathrm{WHO}$ Collaborating Center for Tropical Diseases, 200025 Shanghai, China.

\section{Received: 30 January 2017 Accepted: 1 June 2017}

Published online: 06 July 2017

\section{References}

1. Sachs J, Malaney P. The economic and social burden of malaria. Nature. 2002;415(6872):680-5.

2. Gething PW, Elyazar IR, Moyes CL, Smith DL, Battle KE, Guerra CA, Patil AP, Tatem AJ, Howes RE, Myers MF, George DB, Horby P, Wertheim HF, Price RN, Müeller I, Baird JK, Hay SI. A long neglected world malaria map Plasmodium vivax endemicity in 2010. PLoS Negl Trop Dis. 2012;6(9):e1814

3. WHO. WHO Global Malaria Programme: World Malaria Report. 2015. http://www.who.int/malaria/publications/world_malaria_report_2014/ en/. Accessed 20 Nov 2016.

4. Sharp BL, Kleinschmidt I, Streat E, Maharaj R, Barnes Kl, Durrheim DN, Ridl FC, Morris N, Seocharan I, Kunene S, Grange J, Mthembu JD, Maartens F, Martin CL, Barreto A. Seven years of regional malaria control collaboration-Mozambique, South Africa, and Swaziland. Am J Trop Med Hyg. 2007;76:42-7.

5. Wangdi K, Gatton ML, Kelly GC, Clements AC. Cross-border malaria: a major obstacle for malaria elimination. Adv Parasit. 2015;89:79-107.

6. Cui L, Yan G, Sattabongkot J, Cao Y, Chen B, Che nX, Fan Q, Fang Q, Jongwutiwes S, Parker D, Sirichaisinthop J, Kyaw MP, Su XZ, Yang H, Yang Z, Wang B, Xu J, Zheng B, Zhong D, Zhou G. Malaria in the Greater Mekong Subregion: heterogeneity and complexity. Acta Trop. 2012;121(3):227-39.

7. Stoddard ST, Morrison AC, Vazquez-Prokopec GM, Soldan VP, Kochel TJ, Kitron U, Elder JP, Scott TW. The role of human movement in the transmission of vector-borne pathogens. PLoS Negl Trop Dis. 2009; 3(7):e481.

8. Martens P, Hall L. Malaria on the move: human population movement and malaria transmission. Emerg Infect Dis. 2000;6(2):103.

9. Tatem AJ, Smith DL. International population movements and regional Plasmodium falciparum malaria elimination strategies. Proc Natl Acad Sci U S A. 2010;107(27):12222-7.

10. Wesolowski A, Eagle N, Tatem AJ, Smith DL, Noor AM, Snow RW, Buckee CO. Quantifying the impact of human mobility on malaria. Science. 2012;338(6):267-70.

11. The malERA Consultative Group on Health Systems and Operational Research. A research agenda for malaria eradication: health systems and operational research. PLoS Med. 2011:8:000397.

12. Yin JH, Zhou SS, Xia ZG, Wang RB, Qian YJ, Yang WZ, Zhou XN. Historical patterns of malaria transmission in China. Adv Parasit. 2014:86:1-19.

13. Hu T, Liu YB, Zhang SS, Xia ZG, Zhou SS, Yan J, Cao J, Feng ZC. Shrinking the malaria map in China: measuring the progress of the National Malaria Elimination Programme. Infect Dis Poverty. 2016;5:1.
14. Zeng XC, Sun XD, Li JX, Chen MN, Deng DW, Zhang CL, Lin ZR, Zhou ZY, Zhou YW, Yang YM, Zhou S. Assessment of malaria control consultation and service posts in Yunnan, PR China. Infect Dis Poverty. 2016;5:102.

15. Xia ZG, Yang M, Zhou SS. Malaria situation in the People's Republic of China in 2011. Zhongguo Ji Sheng Chong Xue Yu Ji Sheng Chong Bing Za Zhi. 2012:30(6):419-22. (in Chinese).

16. Zhang L, Feng J, Xia ZG. Malaria situation in the People's Republic of China in 2013. Zhongguo Ji Sheng Chong Xue Yu Ji Sheng Chong Bing Za Zhi. 2014;32(6):407-13. (in Chinese)

17. Bi Y, Tong S. Poverty and malaria in the Yunnan province, China. Infect Dis Poverty. 2014;3:1.

18. Zhou XN, Xia ZG, Wang RB, Qian YJ, Zhou SS, Utzinger J, Tanner M, Kramer R, Yang WZ. Feasibility and roadmap analysis for malaria elimination in China. Adv Parasit. 2014;86:21-46.

19. Xu JW, Li Y, Yang HL, Zhang J, Zhang ZX, Yang YM, Zhou HN, Havumaki J, Li HX, Liu H, Zhou H, Xie XY, Dong JX, Zhang Y, Sun XY, Li B, Li JY, Tian YH, Wang PY, Li BF. Malaria control along China-Myanmar Border during 2007-2013: an integrated impact evaluation. Infect Dis Poverty. 2016:5:75.

20. Xu JW, Liu H. The relationship of malaria between Chinese side and Myanmar's five special regions along China-Myanmar border: a linear regression analysis. Malaria J. 2016;15:368.

21. Eckhoff PA. A malaria transmission-directed model of mosquito life cycle and ecology. Malaria J. 2011;10:e303.

22. Paaijmans KP, Blanford S, Bell AS, Blanford JI, Read AF, Thomas MB. Influence of climate on malaria transmission depends on daily temperature variation. Proc Natl Acad Sci U S A. 2010;107(34):15135-9.

23. Gething PW, Van Boeckel TP, Smith DL, Guerra CA, Patil AP, Snow RW Hay SI. Modelling the global constraints of temperature on transmission of Plasmodium falciparum and P. vivax. Parasite Vector. 2011:4(92):1-11.

24. Ceccato P, Vancutsem C, Klaver R, Rowland J, Connor SJ. A vectorial capacity product to monitor changing malaria transmission potential in epidemic regions of Africa. J Trop Med. 2012. doi:10.1155/2012/595948.

25. Mandal S, Sarkar RR, Sinha S. Mathematical models of malaria-a review. Malaria J. 2011;10:1.

26. China information system for disease control and prevention. http:// www chinacdc.cn/. Accessed 20 Nov 2016

27. Shi B, Liu J, Zhou XN, Yang GJ. Inferring Plasmodium vivax transmission networks from tempo-spatial surveillance data. PLoS Negl Trop Dis. 2014;8(2):e2682.

28. Shi B, Tan Q, Zhou XN, Liu J. Mining geographic variations of Plasmodium vivax for active surveillance: a case study in China. Malaria J. 2015;14:216

29. Macdonald G. Theory of the eradication of malaria. Bull World Health Organ. 1956;15:369-87.

30. Cohen JM, Smith DL, Cotter C, Ward A, Yamey G, Sabot OJ, Moonen B. Malaria resurgence: a systematic review and assessment of its causes. Malaria J. 2012;11:e122.

31. Bruce-Chwatt LJ. Malaria and its control: present situation and future prospects. Annu Rev Publ Health. 1987:8:75-110.

32. Pindolia DK, Garcia AJ, Wesolowski A, Smith DL, Buckee CO, Noor AM, Snow RW, Tatem AJ. Human movement data for malaria control and elimination strategic planning. Malaria J. 2012;11:205

33. Menach AL, Tatem AJ, Cohen JM, Hay SI, Randell H, Patil AP, Smith DL. Travel risk, malaria importation and malaria transmission in Zanzibar. Sci Rep. 2011;1:93

34. Tatem AJ, Huang Z, Narib C, Kumar U, Kandula D, Pindolia DK, Smith DL, Cohen JM, Graupe B, Uusiku P, Lourenço C. Integrating rapid risk mapping and mobile phone call record data for strategic malaria elimination planning. Malaria J. 2014;13:52

35. Pindolia DK, Garcia AJ, Huang Z, Smith DL, Alegana VA, Noor AM, Snow RW, Tatem AJ. The demographics of human and malaria movement and migration patterns in East Africa. Malaria J. 2013;12:397

36. Wang RB, Zhang J, Zhang QF. Malaria baseline survey in four special regions of northern Myanmar near China: a cross-sectional study. Malaria J. 2014;13:302. 